\title{
Successful treatment of post-influenza pseudomembranous necrotising bronchial aspergillosis with liposomal amphotericin, inhaled amphotericin B, gamma interferon and GM-CSF
}

\author{
R J Boots, D L Paterson, A M Allworth, J L Faoagali
}

\begin{abstract}
A case of aspergillus tracheobronchitis following influenza $A$ infection in an immunocompetent 35 year old woman is described that required prolonged mechanical ventilation for airways obstruction. Treatment included liposomal amphotericin, inhaled amphotericin, gamma interferon and GM-CSF. Liposomal amphotericin therapy was associated with reversible hepatosplenomegaly. Inhaled corticosteroids with continued antifungal therapy were used for the management of severe recurrent airway obstruction. After a prolonged course of treatment she survived with fixed airways obstruction unresponsive to corticosteroids.

(Thorax 1999;54:1047-1049)
\end{abstract}

Keywords: Aspergillus; tracheobronchitis; influenza

A 35 year old registered nurse presented to hospital following a flu-like illness with increasing shortness of breath, non-productive cough, central chest pain, and wheeze. She was afebrile and in respiratory distress with widespread expiratory wheezes and patchy inspiratory crackles.

She had been previously healthy. She lived on a rural property but kept no animals. There was no history of previous respiratory disease nor overseas travel. She took no regular medication and weighed $55 \mathrm{~kg}$.

The chest radiograph at presentation was hyperinflated. The $\mathrm{FEV}_{1} / \mathrm{FVC}$ was $1.01 / 1.72$ $(2.53 / 2.93)$ and the $\mathrm{PaO}_{2}$ was $48 \mathrm{~mm} \mathrm{Hg}$ $(6.4 \mathrm{kPa})$ on room air. She had a blood lymphocyte count of $0.30 \times 10^{9} / 1$ (normal range 1.0-4.0) with a neutrophil count of $6.12 \times 10^{9} / 1$ (normal range 2-7.5).

The presumptive diagnosis was asthma and intravenous antibiotics, bronchodilators, and oral prednisone in a dose of $50 \mathrm{mg} /$ day were commenced. Her condition deteriorated with bilateral perihilar alveolar infiltrates on the chest radiograph. She was intubated and mechanical ventilation commenced. She was difficult to hand ventilate and remained hypoxic despite $100 \%$ oxygen.
Bronchoscopic examination revealed thick mucoid inspissated secretions in the trachea and main bronchi. After the airways were cleared a white thick adherent membrane was present in the trachea and the main and segmental bronchi, extending beyond the vision of the bronchoscope. Attempts to remove the membrane resulted in bleeding. Amphotericin B $40 \mathrm{mg} /$ day, intravenous flucytosine $2500 \mathrm{mg}$ six hourly, and nasogastric itraconazole $200 \mathrm{mg}$ eight hourly were commenced. Progressive improvement in the gas exchange was seen over the next eight hours with pressure control ventilation, continuous nebulised salbutamol, and four hourly ipratropium bromide. Corticosteroid treatment was discontinued.

Histological examination of the endobronchial biopsy specimen showed necrotic debris, fibrinopurulent exudate, acutely inflamed bronchial mucosa, squamous metaplasia with marked reactive atypia and small numbers of fungal hyphae. The endobronchial biopsy specimens and bronchoalveolar lavage fluid grew Aspergillus niger. Viral cultures were negative. Paired serum samples for influenza A were positive $>1: 512$. Serological examination for other respiratory viruses was negative.

Ten days after admission to hospital the membrane persisted only in the segmental bronchi of all lobes. Aspergillus niger was again isolated from the bronchial washings. Liposomal amphotericin (Ambisome Faulding and Co Ltd) was given at a dose of $250 \mathrm{mg} /$ day for deteriorating renal function and nebulised amphotericin B, $10 \mathrm{mg}$ twice daily, was added.

Hepatosplenomegaly was noted on the 15 th hospital day which progressed to a liver span of $25 \mathrm{~cm}$ and spleen span of $20 \mathrm{~cm}$ by day 24 . Liver function tests were mildly abnormal. Hepatitis B and C serology were negative and cultures from an open liver biopsy specimen were also negative. Histological examination of the biopsy specimen showed a non-specific chronic hepatitis of moderate severity with focal aggregates of mononuclear cells within the acinar tissue and normal liver architecture. No fungi were seen. Flucytosine and itraconazole treatment were discontinued. 


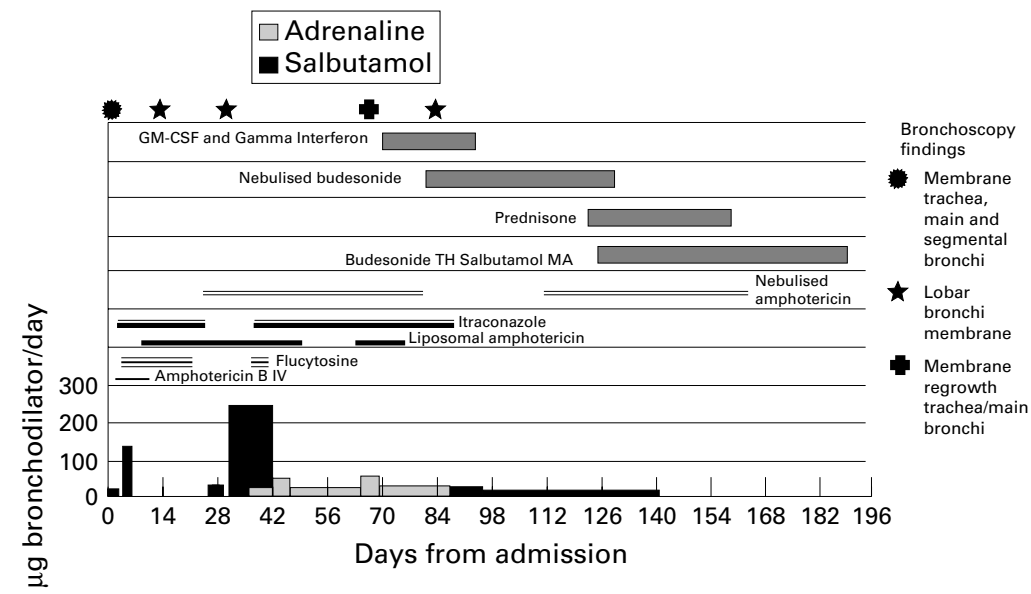

Figure 1 Chart of antifungal and cytokine therapy.

Severe airways obstruction recurred on day 37. There was persisting membrane in the segmental bronchi of all lobes. Nebulised amphotericin was ceased due to concerns that it was contributing to the bronchospasm. Pressure control ventilation required inflation pressures up to $90 \mathrm{~cm} \mathrm{H}_{2} \mathrm{O}$. The patient was hypercapnic to a peak $\mathrm{PaCO}_{2}$ of $90 \mathrm{~mm} \mathrm{Hg}(12 \mathrm{kPa})$. Continuous nebulised adrenaline, aminophylline, ketamine by infusion, and isofluorane by vaporiser had no significant effect on her condition. There was little improvement to day 40 when itraconazole and nebulised amphotericin were recommenced. Sedation and paralysis were stopped by day 52 . Bronchoscopic examination showed membrane in the subsegmental bronchi only. Liposomal amphotericin was discontinued after 42 days and treatment with nebulised amphotericin and itraconazole were continued. There was a slow general improvement to day 58 with a reduction in $\mathrm{FiO}_{2}$ to 0.35 . The hepatomegaly decreased to $12 \mathrm{~cm}$ and the splenomegaly to $9 \mathrm{~cm}$.

From day 65 the course was complicated by episodic severe bronchospasm requiring reintroduction of sedation, paralysis, and pressure control ventilation. Bronchoscopic examination revealed a thick craggy membrane in the distal tracheal and lower airways with cobble stoning of the mucosa. There was persisting lymphopenia of $0.68 \times 10^{9} / 1$. Further biopsy specimens were consistent with an inflammatory membrane but no fungus was seen nor grown. GM-CSF $400 \mu \mathrm{g}$ daily was given subcutaneously for 10 days (Leukomax; Sandoz Australia Pty Ltd) and gamma interferon $200 \mu \mathrm{g}$ subcutaneously (Imukin; Boehinger Ingelheim Pty Ltd) was given three times a week for two weeks. Liposomal amphotericin and continuous nebulised adrenaline were reinstituted and nebulised budesonide commenced.

The patient's condition gradually improved. The hepatosplenomegaly recurred to a liver span of $24 \mathrm{~cm}$ and a spleen span of $22 \mathrm{~cm}$. Liposomal amphotericin was discontinued on day 81 . The lymphocyte count returned to normal by day 122 . By day 131 the liver span had decreased to $18 \mathrm{~cm}$ and the spleen to $6 \mathrm{~cm}$.
Lymphocyte function tests on the fifth hospital day were consistent with generalised $\mathrm{T}$ cell deficiency with poor mitogen responsiveness: CD4 $0.16 \times 10^{9} / 1$ (normal range 0.57$1.67 \times 10^{9} / 1$ ), CD8 $0.09 \times 10^{9} / 1$ (normal range $\left.0.07-0.53 \times 10^{9} / 1\right)$, CD $190.04 \times 10^{9} / 1$ (normal range $\left.0.02-0.43 \times 10^{9} / 1\right)$. HIV serological tests by ELISA were negative. The $\mathrm{T}$ cell deficiency persisted until day 107 after presentation. Lymphocyte reactivity to phytohaemagglutinin, concanavulin A, OKT3, pokeweed mitogen, Candida and Aspergillus antigens was absent on day 12 and returned to normal by day 35. Mantoux testing using avian and human tuberculin were negative despite previous BCG vaccination. The Mantoux test became reactive at $6 \mathrm{~mm}$ by day 131. She remained anergic to tetanus, diphtheria, Streptococcus, Trychophyton, and Proteus despite previous tetanus and diphtheria vaccination. During the first week of admission and again after four and six months immediate, and delayed skin prick tests to Aspergillus niger, Aspergillus fumigatus, and Aspergillus terreus remained nonreactive. A rise in titre to Aspergillus IgM antibody from 2560 to 5120 occurred. There was no IgG response detectable up to day 190 . The appearance of a bone marrow biopsy specimen was consistent with increased myelopoiesis. Antinuclear and rheumatoid factors were not detected and serum immunoglobulins including IgG subclasses were normal.

Due to persisting airways obstruction $60 \mathrm{mg}$ prednisone per day was commenced on day 124 with nebulised amphotericin as a prophylactic. The patient was weaned off the prednisone after one month with no measurable improvement in lung function. A high resolution CT scan of the chest revealed patchy linear shadowing throughout both lung fields and peribronchial thickening with mild central bronchiectasis. She was transferred to a general ward on day 95 and discharged home with independence on day 138. At discharge spirometric values were $\mathrm{FEV}_{1} 1.041$ and FVC 1.891 . She continues well three years later with spirometric values unchanged from discharge. She has returned to employment as a critical care nurse. The clinical course of antifungal and immunotherapy are set out in fig 1 .

\section{Discussion}

This case demonstrates a prolonged persistence of the endobronchial membrane of aspergillus tracheobronchitis for more than 64 days despite antifungal and immunomodulation therapy. The expected duration of an airway membrane in this disease is not described. Host immune status determines the manifestations of aspergillus lung disease and the response to treatment. Patients with aspergillus tracheobronchitis may have less neutropenia and less exposure to corticosteroids and cytotoxic drugs than patients with parenchymal involvement. ${ }^{1}$ The $\mathrm{T}$ cell abnormality may have contributed to this, although aspergillus pulmonary infection is more typical of neutrophil dysfunction or neutropenia. Two forms of fungal tracheobronchitis due to Aspergillus have been described. ${ }^{2}$ Growth can 
be intraluminal with superficial mucosal invasion; a fibrinopurulent membrane is prominent with fungal plugs occluding the bronchial tree. In the absence of parenchymal disease the chest radiograph will be normal. The second manifestation comprises discrete plaques over a small portion of the tracheobronchial tree. Focal pneumonia and abscess formation may result from direct extension.

Our patient had cutaneous anergy and lymphopenia involving $\mathrm{T}$ cells and NK cells but not $\mathrm{B}$ cells. This has been previously described with influenza A infection. ${ }^{3}$ Although there was no clear seroconversion or diagnostic rise in antibodies, the high antibody titre in the absence of vaccination suggested recent influenza infection. Persisting lymphopenia may have been a result of the treatment.

All previously reported cases of postinfluenza aspergillus lung disease have died despite amphotericin treatment. ${ }^{4}$ The airways obstruction in this case was not clinically responsive to high dose inhaled and intravenous bronchodilators. After prolonged treatment with antifungal agents, recurrent airways obstruction resolved with inhaled corticosteroids while antifungal therapy was maintained. GM-CSF can enhance macrophage antifungal activity and stimulate neutrophil function. ${ }^{5}$ Gamma interferon promotes macrophage and NK cell fungal killing. ${ }^{6}$ The relative contributions of antifungal and cytokine therapy in this patient are uncertain.

Nebulised amphotericin has been used both prophylactically and therapeutically for aspergillus lung disease, including aspergillus laryngotracheobronchitis. ${ }^{7}$ It is generally well tolerated but may be associated with significant bronchospasm. ${ }^{8}$ Its contribution to airway obstruction is unclear as withdrawal and subsequent reintroduction did not result in recurrence of the bronchospasm. Liposomal amphotericin has the advantage of being able to be used at a higher dosage with a decreased incidence of renal failure. Hepatosplenomegaly occurred in our patient which resolved after withdrawal of liposomal amphotericin and recurred with its reintroduction. This was associated with a mild hepatitis only. This has not been previously reported. Itraconazole is well described in the primary and adjunctive treatment of fungal infection. In critically ill patients the gastrointestinal absorption of medication is uncertain. Enteral feeding and the common use of stress ulcer prophylaxis may also interfere with the bioavailability of azole based antifungal therapy in this situation. ${ }^{9}$ Antagonism may occur between concurrent amphotericin B and itraconazole therapy. ${ }^{10}$

Reactive airways disease during the acute illness has not been described in tracheobronchitis due to aspergillus. Fixed airway obstruction has persisted despite inhaled and systemic steroid trials followed for over three years. This case resulted in survival with an excellent functional outcome in a patient with aspergillus tracheobronchitis following influenza infection. Intensive support along with multimodality treatment may have a place in the management of these patients.

1 Angelucci E, Ugolini M, Lucarelli G, et al. Endobronchial aspergillosis in marrow transplant patients. Bone Marrow Transplant 1991;8:328-9.

2 Clarke A, Skelton J, Fraser RS. Fungal tracheobronchitis. Report of 9 cases and review of the literature. Medicine 1991;70:1-14.

3 Criswell BS, Couch RB, Greenberg SB, et al. The lymphocyte response to influenza in humans. Am Rev Respir Dis 1979;120:700-3.

4 Jariwalla AG, Smith AP, Melville-Jones G. Necrotising aspergillosis complicating fulminating viral pneumonia. Thorax 1980;35:215-6.

5 Bodey GP, Anaissie E, Gutterman J, et al. Role of granulocyte-macrophage colony stimulating factor as adjuvant treatment in neutropenic patients with bacterial and fungal infection. Eur 7 Clin Microbiol Infect Dis 1994;13: S18-22.

6 Murphy JW. Immunity to fungi. Curr Opin Immunol 1990;2: 360-7.

7 Dal Conte I, Riva G, Obert R, et al. Tracheobronchial aspergillosis in a patient with AIDS treated with aerosolized amphotericin B combined with itraconazole. Mycoses 1996;39:371-4.

8 Gryn J, Goldberg J, Johnson E, et al. The toxicity of daily inhaled amphotericin B. Am f Clin Oncol 1993;16:43-6.

9 Puttick MPE, Phillips P. Itraconazole: precautions regarding drug interactions and bioavailability. Can f Infect Dis 1994; 5:179-83.

10 Schaffer A, Bohler A. Amphotericin B refractory aspergillosis after itraconazole: evidence for significant antagonism Mycoses 1993;36:421-4. 